\title{
TCP Wave estimation of the optimal operating point using ACK trains
}

\author{
A. Abdelsalam, C. Roseti, and F. Zampognaro \\ University of Rome "Tor Vergata" \\ Via del Politecnico 1, 00133 \\ Rome, Italy
}

\author{
N. Patriciello \\ Centre Tecnològic de Telecomunicacions de Catalunya \\ Av. Carl Friedrich Gauss 7, 08860 \\ Castelldefels, Barcelona, Spain
}

- Low occurrence of link transmission errors, so that all losses are most likely due to congestion (justifying the $C W N D$ reduction).

\begin{abstract}
TCP Wave changes the typical TCP transmission paradigm by replacing the ACK-clocked sliding window with selfscheduled bursts. In response to bursts, unmodified TCP receivers generate ACK trains, which carry useful information about the end-to-end link characteristics. TCP Wave inspects ACK-train flow to measure the following parameters: (i) ACK train spread (namely ACK train dispersion) and (ii) RTT variations. The former is expected to provide the overall "service capacity", meant as the maximum capacity allowed over the end-to-end path, while the latter is considered as a congestion indicator. The joint use of such measurements allows TCP Wave to fine-tune the transmission rate to accurately match current network resource availability in the bottleneck link. This paper analysis TCP Wave ACK train-based measurements on a broad set of simulated links compliant to characteristics of today's real networks. To this scope, a testbed with TCP Wave implementation on Linux OS is used to perform tests varying both bottleneck capacity and physical latency.
\end{abstract}

Keywords—TCP Wave; bandwidth estimation; Linux; bottleneck

\section{DESCRIPTION AND LIMITATIONS OF TCP CONGESTION} CONTROL

In traditional TCP [1], Congestion Window (CWND) value updates and scheduling of new segments to be transmitted on the network are triggered upon reception of ACKs, making TCP transmission in fact ACK-clocked. On the other hand, ACK reception timing is depending on the Round-Trip Time (RTT), computed at the sender, as the time interval between a packet sending and the reception of the corresponding Acknowledgement (ACK). Thus, the higher is the RTT, the slower is the triggering of TCP rate adaptations, which can be realized by different congestion control algorithms such as New Reno, Cubic, Vegas, Westwood, etc. Furthermore, TCP generally follows a reactive strategy for the congestion control: wait for the trouble and then solve it. Congestion control algorithms progressively increase $C W N D$ upon ACK receptions and then reduce it in case of loss detections (through duplicate ACK receptions or Retransmission Time Out expiration RTO). In both cases, operation timing is entirely relying on RTT. The resulting protocol was performing quite good in $80 \mathrm{~s}-90 \mathrm{~s}$ wired networks characterized by:

- $\quad$ Low RTT, allowing fast actions on $C W N D$;

- Relatively small bandwidth (BW) at the bottleneck, keeping small the $C W N D$ value to achieve maximum rate (provided by the BW·RTT product).

- Small bottleneck buffers, limiting the RTT upper bound;
- Long transfers, providing time to achieve TCP steadystate.

Such a communication environment went through significant changes over the years, with the continuous increase in link bandwidth, the proliferation of wireless links and/or lossy links (with transmission errors) [2][3], the higher in-network buffering capability [4][5], and the deployment of hybrid networks with variable topologies and physical characteristics [6]. Besides, HTTP traffic dominance led to frequent short TCP steady-state. All the above factors contribute to break original TCP design assumptions leading up to significantly impaired performance. Consequentially, protocol enhancements and modifications became needed with the aim to allow transmissions at the maximum eligible rate (optimal bottleneck utilization) without significant increases on the end-to-end RTT (no congestion and flow fairness). Such a condition is referred as optimal operating point and cannot be achieved by traditional $C W N D$-based and ACK-clocked TCP paradigm, because it leverages on the RTT only. In fact, the convergence to the optimal operating point is a problem with two-variables: the Bottleneck Capacity (BC) and the Congestion Level (CL). A single sender-side measurement, such as the RTT, is then not sufficient to assess the optimal transmission settings. For instance, RTT increases can be jointly or alternatively due to both a rate higher than exceeding and CL increases (more competitive traffic), and it is quite challenging to solve this ambiguity. An additional sender-side measurement is then necessary to solve this problem. At the same time, another limitation can derive from the ACK-clocked operations of standard TCP, which limit the reactiveness to changes being proportional to the RTT.

TCP Wave is a recent alternative transport protocol that pursues by design the objective to work around the optimal operating point, by leveraging on two "not-correlated" senderside measurements, which are allowed by a new transmission paradigm based on bursts (which are not ACK-clocked). In fact, transmission in bursts allows the generation of so-called $A C K$ trains, which carry, together with traditional RTT information, the ACK train dispersion over time, strictly correlated to the BC. TCP Wave modifies the congestion control mechanisms, without requiring neither ad-hoc methods at the lower layers (i.e., ad-hoc packet scheduler) nor receiver changes. Therefore, transfers [7], usually terminated before the achievement of the 
it can constitute a promising new technology once demonstrated the validity and consistency of the performed measurements over a broad range of network configurations.

In this framework, the scope of this paper is to validate the TCP Wave measurements with respect to the expected (ideal) values, using a real protocol implementation on Linux kernel [8]. Specifically, Section II provides a general overview of TCP Wave aimed to highlight how ACK-based measurement impact on protocol functions; Section III details the method to take the target measurements and how they are used to compute a suitable TxTime applied for burst transmission; Section IV presents result of a test campaign tailored to the verification of the measurement validity with some preliminary performance results, postponing a full characterization of the rate control performance in future works.

\section{TCP WAVE: PROTOCOL DESCRIPTION}

TCP Wave is a transport protocol designed as an alternative to traditional $C W N D$-based TCP versions for current broadband networks. A full protocol specification is provided in [9], while performance assessments under various simulated network and application configurations are also studied in [10][11][12][13].

The main innovative protocol characteristics can be summarized as follows:

- Burst transmission paradigm;

- $\quad$ A transport layer self-managed clock at the TCP sender;

- Proactive rate control.

The joint effect of such characteristics leads to a reliable transport protocol radically different from traditional TCP design, while still guaranteeing the fundamental TCP properties: congestion control and avoidance, flow control, error control, inter-flow fairness and friendliness. Therefore, TCP Wave is not an additional TCP enhanced version, but it proposes a reviewed transmission paradigm compliant to the fundamental TCP principles. At the same time, the transport layer receiver does not need any modification, making possible a fast deployment of the new protocol in co-existence with current TCP implementations.

The fundamental principle of TCP Wave is the packet transmission in bursts adequately spaced each other. Thus, scheduled transmissions envisage bursts of BURST packets, with a default value BURST $_{0}$ of 10 packets (used as the initial value and as a target value during the connection). In practice, TCP Wave sender increases the CWND by BURST upon the expiration of an internal timer, which has an expiration time equal to a value called TxTime, so that the actual transmission rate is given by:

$$
\text { TxRate }=\frac{\text { BURST }}{\text { TxTime }}
$$

Then, TCP Wave transmission rate is fully regulated by the current TxTime value, while ACK reception does not trigger any direct action on the CWND. TxTime determines the actual rate and its value is dynamically updated in accordance with both $B C$ and $C L$ measurements. In particular, updated TxTime values are computed by an ACK-based Capacity and Congestion Estimation (ACCE) component that continuously monitors the received ACK flow with the aim to measure two parameters: the
RTT (indicator for CL) and the ACK train dispersion (indicator for $B C$ ).

Waiting for the reception of the first ACK train (corresponding to a full BURST transmission), TxTime is set to a default value (TxTime 0 ), which determines the rate during the initial blind phase. Most likely, such a phase lasts 1 RTT only. Recommendations to define target $\mathrm{BURST}_{0}$ foster the achievement of the most of shortest transfer (i.e., messaging-like exchanges) within the first burst, while TxTime 0 is set to guarantee a minimum transmission rate to short transfers needing more than one burst, but terminating before the reception of the first ACK train.

Another key change introduced by TCP Wave concerns the attempt to strictly follow the network status and set the transmission rate to prevent congestion. TCP Wave logic is to transmit "as much as possible" without inflating the RTT, then constantly working around the optimal operating point. This is in contrast with the traditional TCP "reactive" philosophy: "send even more packets until losses are detected, then repair from both losses and injected congestion".

Upon the reception of the first ACK train, TCP Wave enters in the Tracking Mode, in which the TxTime is updated by any further ACK train reception using the following equation:

$$
\text { TxTime }_{n}=\text { ACK_Train_Disp } n+0.5 \cdot \Delta R T T_{n} \quad \text { [Eq. 2] }
$$

where $n$ indicates the ACK train sequence number. The ACK train dispersion factor (ACK_Train_Disp) indicates an assessment of the TxTime to achieve $B C$, while $0.5 \cdot \Delta R T T_{n}$ is a correction factor that takes into account $C L$. In more details, $\Delta R T T_{n}$ is the difference between the current average $R T T_{n}$ and the minimum measured RTT $\left(R T T_{\min }\right)$. The 0.5 multiplier factor is to equally split the charge for the congestion recovery between the target TCP Wave connection and the competing flows. In theory, a TCP Wave connection should run in Tracking Mode for its whole lifetime. A $\triangle R T T$ threshold, namely $\beta$, is defined to take into account situations where RTT increase is significant, despite the continuous rate adaptation following [Eq. 2]. A significant increase can occur in various circumstances: greedy competing traffic (on top of different transport protocols, i.e., UDP), network changes, an unusual high number of simultaneous flows creating a temporary congestion. When $\Delta R T T_{n}>\beta$, TCP Wave triggers Adjustment mode that performs a reset of both the transmission rate (reverting TxTime to TxTimeo) and the $R T T_{\min }$ reference. The rationale is to temporary transmit at a minimum rate and sense if something is changed in the network by looking at new ACK trains. In order to skip the information coming from the data in-flight when the algorithm enters Adjustment Mode, a "Stability Factor" value is calculated, following [Eq. 3].

StabilityFactor $=\frac{R T T_{n}}{\text { TxTime }_{n}}$

In this way, a new TxTime update, which is the event to go back in Tracking Mode, is computed after skipping an upcoming number of ACK trains equal to Stability Factor. As a consequence, the duration of Adjustment Mode is roughly $R T T_{n}$. 


\section{ACCE MEASUREMENTS}

TCP Wave operations leverage the assumption that measurements provided by the $A C C E$ component are reliable and accurately reflect actual $B C$ and $C L$. This requires that $A C K$ Train Dispersion reflects the ideal BURST spacing due to the bottleneck [14] for the achievement of a transmission rate equal to BC, and the RTT increases reflect the actual congestion status filtering possible spare traffic spikes, the effects of possible retransmissions, cumulative $\mathrm{ACKs}, \mathrm{ACK} /$ packet aggregation, and so on. Therefore, procedures and algorithms applied in taking the measurements are of paramount importance.

\section{A. ACK flow handling}

$A C C E$ monitors the whole ACK flow to compute target statistics: the RTT of the first packet of each burst, namely $R T T_{\text {pilot }}$, and the time between last (last_ack_time) and first ACK (first_ack time) of a train. As a reference, it has access to the burst history queue that records the size of in-flight bursts (transmitted and waiting to receive the corresponding ACK train). As soon as a burst is fully acknowledged, a measurement round is completed and the corresponding record is deleted from the burst history queue. Therefore, the first-placed record into the queue provides to $A C C E$ the boundaries of the current ACK train.

Basically, a new ACK can trigger a set of operations associated to three logic states managed by the ACCE algorithm:

- Round begin - it is triggered when ACK is believed as the first ACK of a train. This ACK is used to compute the RTT value associated to the whole burst $\left(R T T_{\text {pilot }}\right)$ and to set the initial time reference for the current ACK train (first_ack_time).

- Round_middle - it is triggered for each ACK received following the first ACK in the train. It updates both the number of ACKs within the train and the time reference of the last ACK in the train (last_ack_time).

- Round_end-ACCE enters in this state when received ACK completes the currently monitored train. Basically, the ACK updates last_ack time and triggers final calculation of both ACK train dispersion and the RTT values.

Upon an ACK reception, both the number of acknowledged bytes (ACKed bytes) and the RTT (current RTT) are preliminarily examined before entering a logic state. Because a consistent ACK train structure requires more than one ACK per burst, ACCE filters any "big" cumulative ACK that would not allow train-based measurements. When $A C K e d$ bytes are larger than the size of the current burst, ACK train structure is considered broken and any timing parameters previously registered for the current train not consistent. Accordingly, any fully acknowledged burst by such a "big" ACK is cleared from the burst queue, while all the timing information is reset. This reset operation allows the TCP Wave transmission parameters to be updated from the latest correctly computed measurements instead of relying on inconsistent samples. Whereas, if there are no valid measurements previously achieved, the transmission parameters are simply not updated and TxTimes remains effective. Still, the "big" cumulative ACK may also trigger the Round_begin if it partially covers a burst in the queue (newest burst which is partially acknowledged by the ACK), making the ACK as the first one of the new train.

Without TCP losses, ACKs usually cover a number of bytes lower than burst size (TCP receiver triggers one ACK for every two full segments by default [15]), and ACK train structure is kept consistent. Thus, $A C C E$ proceeds by checking if the received ACK is the first one in a train. This is done by checking the first_ack_time value, which is set to the current time by the Round_begin, and then reset to 0 when the Round_end.

Whenever an ACK is not the first of a train, a further check on its timing consistency is performed through the associated RTT value (current RTT). Since a burst transmission envisages the in-order injection of multiple segments at once (same sending time reference), the RTT within an unaltered ACK train must be monotonically growing. On the contrary, a decreased RTT indicates a misalignment between the ACK train and its corresponding burst. Thus, in this case, ACCE enforces a Round begin because it considers the ACK as the first of the train. This occurrence is mostly due to packet transmissions by the underlying operating system out of the TCP Wave control, which create unexpected shifting in the ACK train structure.

In any other case, when ACK is neither marked as first of the train nor presents a decreased RTT, it is assumed to be in the middle of the current train, and therefore Round_middle is triggered. For each Round_middle ACK, ACCE tracks the sum of ACKed bytes by the train. If the ACKed bytes is greater than or equal to corresponding burst size BURST, the ACK is assumed to terminate the train, and the measurement for the current burst is concluded triggering a Round_end. However, within this last state, two sub-cases are possible due to cumulative nature of ACKs: (1) the ACK acknowledges the exact number of segments in the burst, or (2) the ACK also acknowledges some segments of the subsequent burst.

In the first case, information for the number of ACKs in the train, first ack time, and last ack time are used to compute the ACK train dispersion and the RTT measurements for the current train are concluded. Consequently, all the internal ACCE variables are reset and the upcoming $\mathrm{ACK}$ will be accounted as the first of a new train. In the second case, the last ACK terminates the current train but also includes one or more segments of the subsequent burst. This means that the ACK carries timing information inflated by transmission of two consecutive bursts. Thus, ACCE uses this last ACK to terminates the round but utilizing the last_ack_time set by the prior ACK in the previous Round_middle. Furthermore, a Round_begin must be triggered immediately taking into account the last ACK as the first of a new round.

\section{B. Bottleneck capacity and congestion assessment}

At the end of each round, ACCE aims to provide as outputs possible new samples for ACK train dispersion and RTT, feeding TCP Wave algorithms. A new ACK train dispersion sample is computed only if the burst size is equal to the reference burst size $\left(B U R S T_{0}\right)$, because smaller trains could cause two main drawbacks for the measurements:

1. A normalization is required for the final ACK train dispersion sample used to update TxTime, which is always tailored for $B U R S T_{0}$ transmissions; 
2. The smaller is the burst and the lower is the number of ACKs within a train, making the measurements mostly sensitive to timing glitches.

Then, when the size of the current burst is smaller than the $B U R S T_{0}$, ACCE returns the value of the latest valid measurement. However, transmission of burst smaller than $B U R S T_{0}$ can be due to sender buffer limitations, or applicationlimited data at the moment the burst is generated. Similarly, when the number of received ACK in a train is lower than 3 , the ACK train dispersion is not updated.

On the contrary, when the current burst size is equal to $B U R S T_{0}$, a "fresh" ACK train dispersion is computed as follows:

$$
\begin{aligned}
& \text { ACK_Train_Disp } p_{n}= \\
& (\text { last_ack_time-first_ack_time }) \cdot \frac{\text { number acks per train }}{\text { number acks per train }-1}
\end{aligned}
$$

The last term in [Eq. 4] adds a time contribution to be accounted after the last $\mathrm{ACK}$, equal to the average spacing among the consecutive ACK pairs within the train. In fact, last ack time does not include the time needed to process the last segment of the burst. Definitively, the calculated value for the ACK train dispersion is used to update the TxTime value by feeding [Eq. 2].

The RTT measurement function takes as input argument only the $R T T_{\text {pilot }}$ value, computed in the Round_begin. Such an RTT value is representative for the whole burst and it is used to assess congestion status. First, TCP Wave sender updates the minimum RTT experienced overall the current Tracking mode, namely $R T T_{\text {min }}$, as the lowest $R T T_{\text {pilot }}$ value. Additionally, to avoid the effect of spare RTT spikes that may occur due to network dynamics not strictly correlated to the congestion status, a smoothed RTT, namely $R T T_{a v g}$, is computed by using an Exponentially Weighted Moving Average (EDWA) filter:

$$
R T T_{\text {avg }}=\alpha \times R T T_{\text {avg }}+(1-\alpha) \times R T T_{\text {pilot }} \quad \text { [Eq. 5] }
$$

where $\alpha$ is the filter parameter dynamically computed as:

$$
\alpha=\left(R T T_{\text {pilot }}-R T T_{\min }\right) / R T T_{\text {pilot }}
$$

Thus, the larger is the difference between the new $R T T_{\text {pilot }}$ and the $R T T_{\text {min }}$, the stronger is the smoothing effect performed by the $\alpha$ filter.

The final output of the RTT measurement function is the $\triangle R T T$ value:

$$
\Delta R T T=R T T_{a v g}-R T T_{\text {min }}
$$

Such a value feeds [Eq. 2] and then jointly to the computed ack_train_disp, determines the updated TxTime. In addition, $\triangle R T T$ is compared to the $\beta$ threshold, in order to determine the next TCP Wave mode, either Tracking or Adjustment.

\section{ACCE Measurement Validation}

As a target objective, ACCE is required to continuously compute updated estimations of $B C$ in terms of ACK train dispersion and $C L$ in terms of $\triangle R T T$. The higher is the percentage of valid samples, the better is the supported reactivity to network and load changes. Thus, the percentage of
Round_end triggering represents a clear indication of the validity of the proposed scheme for handling ACK flow. In addition, the evaluation of the achieved ACK train dispersion samples with respect to the ideal value is of paramount importance for the measurement reliability. Inconsistent values may lead to wrong assumptions on the underlying network with a harmful impact on the transmission rate. By design, TCP Wave rate control uses $\triangle R T T$ to compensate a possible underestimation of the ACK train dispersion, making somehow the protocol robust to small measurements errors.

As an ideal benchmark, optimum performance for a single TCP Wave flow needs to achieve from ACCE ACK train dispersion such that:

$$
B C=B U R S T /(\text { ack_train_disp })
$$

$$
\text { and } \triangle R T T=0 \text {. }
$$

For this purpose, we built a basic testbed setup, shown in Figure 1, that consists of three Linux machines acting as sender, receiver and bottleneck link emulator, respectively. Each node pair is connected through $1 \mathrm{Gbit} / \mathrm{s}$ network ethernet interface. In the sender machine, we run a modified kernel that contains the latest implementation of TCP Wave [8], while receiver runs a default Linux network configuration. The intermediate node is configured as a Linux based router (ZeroShell) [16] in order to provide a traffic shaping and QoS management able to apply bandwidth and delay constraints.

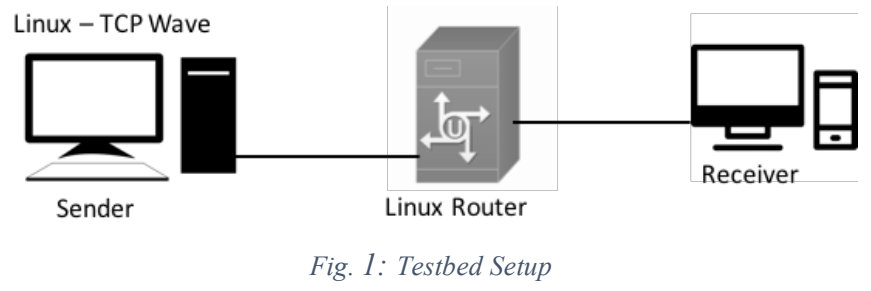

For the sake of an exhaustive test campaign, the bandwidth value is varied in the range [ $2 \mathrm{Mbit} / \mathrm{s}-30 \mathrm{Mbit} / \mathrm{s}$ ], with a 2 $\mathrm{Mbit} / \mathrm{s}$ granularity. Such a range covers most of the current consumer IP connectivity services over public networks. Besides, the physical delay is jointly varied to reproduce four different communication scenarios in terms of end-to-end physical RTT: no extra delay (RTT $\approx 0.3 \mathrm{~ms}$ ), RTT $=100 \mathrm{~ms}$, $\mathrm{RTT}=200 \mathrm{~ms}, \mathrm{RTT}=500 \mathrm{~ms}$. Note that ACK train dispersion should be independent of latency, so that it is worth to verify such a property over a large set of RTT configurations in real environments. Tests consist on running an Iperf [17] transfer of 500 Mbytes over a TCP Wave connection in order to gather a high number of ACK train dispersion samples $(\approx 36 \mathrm{~K})$, allowing to achieve a statistically significant Probability Density Function (PDF). Figure 2 shows the PDF of three selected bandwidth configurations from our test campaign. Figure 2 (a) shows PDF of the ACK train dispersion in case of $B C$ equal to 2 $\mathrm{Mbit} / \mathrm{s}$ in comparison with the optimum value $(60.6 \mathrm{~ms})$ to exactly match $B C$. First, results demonstrate how measurements are resilient to different possible RTT, because of the almost precise overlapping among the various curves. Simulation output reports that the overall percentage of valid samples (triggering a successful Round-end) varies from $80.6 \%$ with $\mathrm{RTT}=500 \mathrm{~ms}$ to $99.9 \%$ with $\mathrm{RTT}=0.3 \mathrm{~ms}$. 


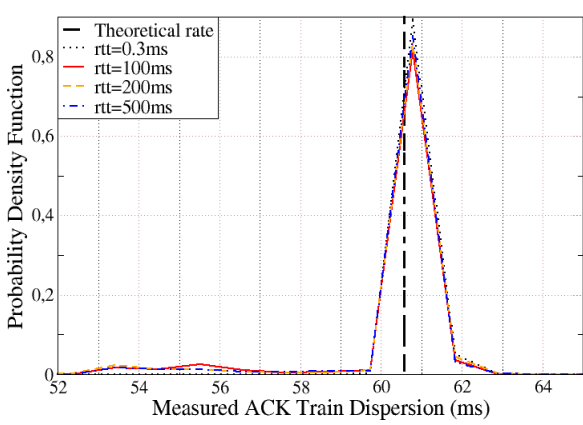

(a) $\mathrm{BC}=2 \mathrm{Mbit} / \mathrm{s}$

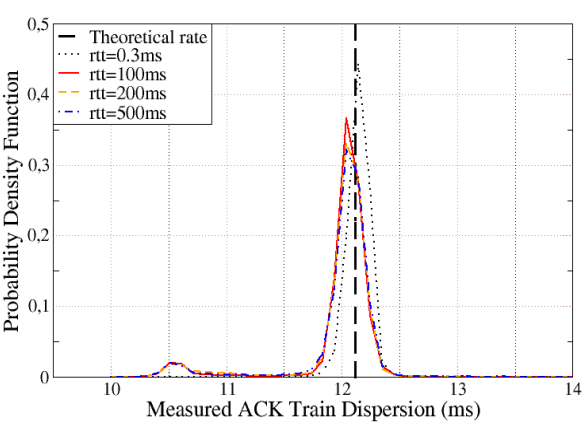

(b) $\mathrm{BC}=10 \mathrm{Mbit} / \mathrm{s}$

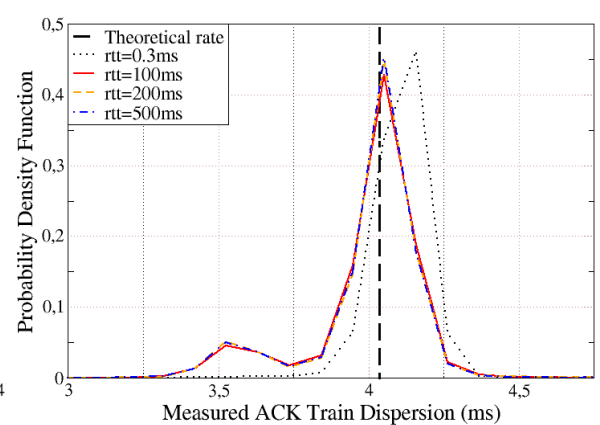

(c) $\mathrm{BC}=30 \mathrm{Mbit} / \mathrm{s}$

Fig. 2: PDF of ACK train dispersion measurements

Therefore, ACCE is able to maintain a continuous synchronization with the ACK train structure efficiently feeding TCP Wave rate control component with ever updated values. Valid measurements fall in more than $80 \%$ very close, while slightly higher, to the optimum value. Definitively, the ACCE outputs strictly reflect $\mathrm{BC}$ and then represent a suitable input for TCP Wave rate control.

Figure 2 (b) concerns the test with $\mathrm{BC}=10 \mathrm{Mbit} / \mathrm{s}$. The number of valid samples is similar to that achieved in the previous case, then confirming a very good synchronization with the ACK train structure. Although the most of the measurement values are contained in a small-time interval around the ideal one (12.11 ms), PDF shape results a bit more spread over such an interval, as remarked by the PDF peak at about 0.35 . This means that ACK train dispersion presents a larger standard deviation from the average value. Finally, PDF with $\mathrm{BC}=30 \mathrm{Mbit} / \mathrm{s}$, in Figure 2 (c), presents similar characteristics and leads to similar considerations as those discussed for $\mathrm{BC}=10 \mathrm{Mbit} / \mathrm{s}$. It is worth to remark that such a high bandwidth implies measurements of much smaller time intervals which are more sensitive to little variations from the ideal value (4.04 ms).

\section{A. Preliminary TCP Wave performance analysis}

Figure 3 summarizes performance achieved by TCP Wave in terms of average bottleneck utilization, during the same simulation runs described before.

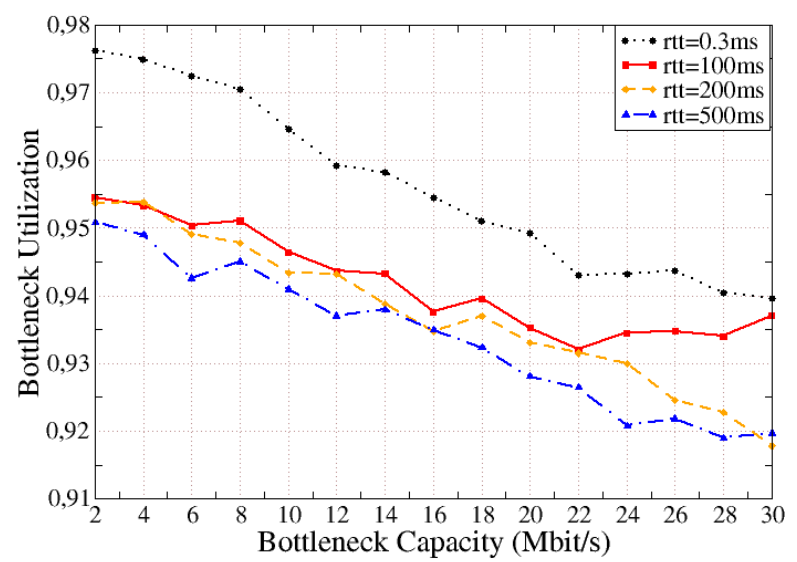

Fig. 3 Measured goodput of TCP Wave over different BDP links
TCP sets transmission rate by updating TxTime as defined by [Eq. 2]. Full utilization $(=1)$ indicates a perfect matching between transmission rate and $\mathrm{BC}$. Results in Figure 3 show a little utilization improvement as the latency decreases. This trend can be explained by the analysis of ACK train dispersion PDF curves that present some small tails at values lower than the average one in case of high RTT. This leads to slightly over transmit and to a proportional increase of RTT, which in turn equalizes the next TxTime update. Such a control loop makes small oscillations of the transmission rate over time (which in fact give the name to the protocol), that result in the marginal utilization decrease. Utilization slightly decreases also for the highest bottleneck capacities. This is due to the handling of smaller ACK train dispersion measurements, for which some little deviations from the ideal value causes a higher rate reduction.

The achieved transmission rate depends on the measured ACK train dispersion as well as the computed $\triangle R T T$ which, for a complete analysis, whose values are reported in Figure 4 for the different configurations. The gathered low $\triangle R T T$ values indicate a transmission rate quite similar to the delivery rate, meant as the actual rate at the receiver, and no major selfinduced congestion to the bottleneck is generated.

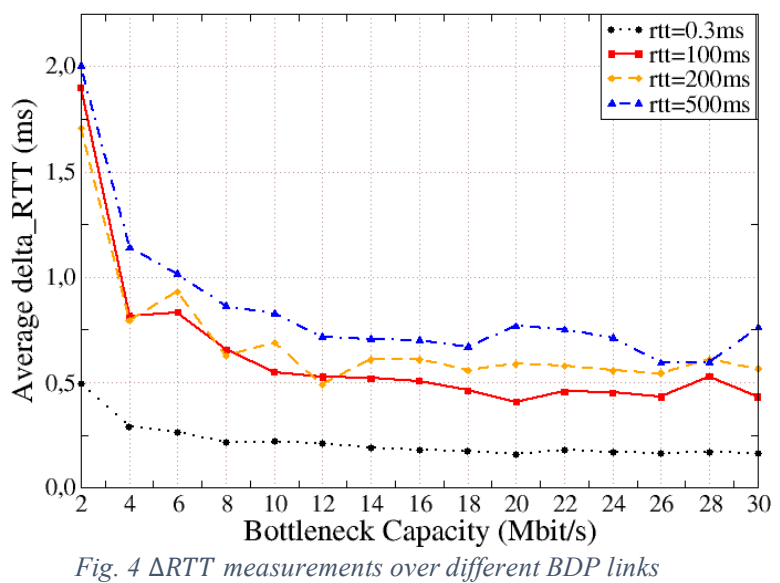

As a summary, overall performance is optimal with a bottleneck utilization always above $92 \%$ and marginal values of $\Delta R T T(<2 \mathrm{~ms})$, which indicate how TCP Wave is able to work 
in the proximity of the optimal operating point over a large range of network configurations

\section{CONCLUSIONS}

This paper investigates the consistency of ACK train-based measurements performed by TCP Wave to update its transmission rate. Tests leveraged a Linux TCP Wave sender implementation, which allowed to interwork with a real TCP receiver, then making ACK generation realistic. Results are very promising with filtered ACK train dispersion samples strictly reflecting the bottleneck capacity and then efficiently exploitable to implement the TCP Wave proactive rate control. Another important achievement has been the demonstration of ACK train dispersion decorrelation from network latency. This property implies that ACK train dispersion and measured RTT can be considered as two independent input parameters, and as such, they can be fruitfully exploited to discriminate the Bottleneck Capacity (BC) from the Congestion Level (CL). Thus, such measurements are currently used by TCP Wave rate control for the achievement of the optimal operating point, as demonstrated by bottleneck utilization jointly to a stable RTT close to its minimum value, as presented in the paper for a large range of bottleneck capacity values.

\section{ACKNOWLEDGEMENTS}

This work was partially funded by Spanish MINECO grant TEC2017-88373-R (5G-REFINE) and Generalitat de Catalunya grant 2017 SGR 1195.

\section{REFERENCES}

[1] M. Allman, V. Paxson, and E. Blanton, “TCP Congestion Control," RFC 5681, IETF, Sep. 2009.

[2] Luglio, M., Roseti, C., Savone, G., \& Zampognaro, F. (2009). Cross-layer architecture for a satellite-wi-fi efficient handover. IEEE Transactions on Vehicular Technology, 58(6), 2990-3001

[3] Bacco, M., Gotta, A., Roseti, C., \& Zampognaro, F. (2014). A study on TCP error recovery interaction with random access satellite schemes. Paper presented at the 2014 7th Advanced Satellite Multimedia Systems Conference and the 13th Signal Processing for Space Communications Workshop, ASMS/SPSC 2014, , 2014-January 405-410.

[4] L. Carniato, F. Fongher, M. Luglio, W. Munarini, C. Roseti, F. Zampognaro, Traffic analysis and network dimensioning through simulation and emulation for Ka band high capacity satellite systems, IEEE 18th International Workshop on Computer Aided Modeling Analysis and Design of Communication Links and Networks (CAMAD), 2013, Pages: $223-227$.

[5] M. Luglio, G. Theodoridis, C. Roseti, N. Pavlidou, A TCP driven CAC scheme: efficient resource utilization in a leaky HAP-Satellite integrated scenario, on IEEE Transactions on Aerospace and Electronic Systems (TAES), Vol. 45, N. 3, pp. 885-898, July 2009.

[6] M. Luglio, C. Roseti, G. Savone, F. Zampognaro, TCP Noordwijk for High-Speed Trains, First International Conference on Advances in Satellite and Space Communications, 2009, SPACOMM 2009, 20-25 July 2009, Page(s): 102-106

[7] Salam, A. A., Luglio, M., Roseti, C., \& Zampognaro, F. (2014). SPDY multiplexing approach on long-latency links. Paper presented at the IEEE Wireless Communications and Networking Conference, WCNC.

[8] TCP Wave source code and installation guide for Linux: accessible online at: http://tlcsat.uniroma2.it/tcp wave4linux

[9] Abdelsalam, A., Luglio, M., Roseti, C. Zampognaro, F., 2017. TCP Wave: A new reliable transport approach for future Internet, Computer Networks, 112, pp.122-143.

[10] A. Abdelsalam, M. Luglio, C. Roseti, F. Zampognaro., TCP Wave Resilience to Link Changes - A New Transport Layer Approach Towards Dynamic Communication Environments, 13th International Joint Conference on e-Business and Telecommunications (DCNET 2016), Lisbon, Portugal, July 2016

[11] Abdelsalam, A., Luglio, M., Roseti, C., Zampognaro, F., A burstapproach for transmission of TCP traffic over DVB-RCS2 links, IEEE 20th International Workshop on Computer Aided Modelling and Design of Communication Links and Networks (CAMAD), 7-9 September 2015, University of Surrey, Guildford, UK, September, 2015, pp. 175-179.

[12] Abdelsalam, A., Roseti, C., Zampognaro, F., 2017. Steady-state performance evaluation of Linux TCPs versus TCP wave over leaky satellite links, China Communications 2017/3, pp.17-30.

[13] Abdelsalam A, Luglio M, Roseti C, Zampognaro F. Evaluation of TCP wave performance applied to real HTTP traffic. In Networks, Computers and Communications (ISNCC), 2017 International Symposium on 2017 May 16 (pp. 1-6). IEEE.

[14] C. Dovrolis, P. Ramanathan and D. Moore, "What do packet dispersion techniques measure?," Proceedings IEEE INFOCOM 2001. Conference on Computer Communications. Twentieth Annual Joint Conference of the IEEE Computer and Communications Society, Anchorage, AK, 2001, pp. 905-914 vol.2.

[15] Allman, Mark, Vern Paxson, and Ethan Blanton. TCP congestion control. No. RFC 5681. 2009.

[16] Linux distribution for networking routers and embedded devices, official web site http://www.zeroshell.org

[17] Open source speed test tool for TCP, UDP and SCTP, official web site https://iperf.fr 